\title{
The temperature - insulative material that can work as the insulation and siding and decorative material
}

\author{
Boris Aksenov ${ }^{1}$, Oleg Stepanov ${ }^{1 *}$, and ludmila Stefurak ${ }^{1}$ \\ ${ }^{1}$ Industrial University of Tyumen, Volodarskogo str. 38, Tyumen, 625001, Russia,
}

\begin{abstract}
The article analyzes the new temperature - insulative materials. It is motivated that the temperature - insulative material capable to work in the panel as a temperature - insulative and facing-decorative material can be only composite type. The composition of the new material, the technology of its production, as well as the mathematical model of the mechanism of formation of strong crusts at its surfaces. The material that is used for the manufacture of roofing panels, panels BKU with an internal non-metallic layer. These panels allow you to break the bridges of cold in butt joints, which improves thermal performance, reliability and durability of the building as a whole. Based on the developed mathematical model, you can predict the properties of foam plastic.
\end{abstract}

\section{Introduction}

The complete-block method of construction became the main one in arrangement of West Siberian deposits [1]. Especially increases the importance of the construction in framepanel version, but with the use of powerful bases with the installed equipment. The crucial feature of such buildings will be thermophysical parameters base and walling.

In the three-layered panels frame type for facing-decoration required ists metal. In the production of double-layer panels, at least twice the metal consumption can be reduced. Double-layer and even" metal-free " panels can be manufactured only on high-strength insulation.

At the existing level of panel production technology, only the thermal insulation properties of foams are useful, such an important indicator as strength is not used. The strength of the panels is provided exclusively by structural elements. For example, the steel frame of the panel is sheathed with steel (aluminum, asbestos cement) sheets and filled with foam or mineral wool plates [10]. This leads to a rise in price of panels and deterioration of their thermal performance. Structurally these disadvantages to offset the difficult.

The development of science and technology of foam plastics production has led to the emergence of new ways to influence the final properties of foam in the process of their formation [11,14]. On the basis of the multidimensional network model, a model of technical evolution of panel samples was developed, which differ from the existing best technical and economic indicators $[1,16,20]$.

As source materials were considered insulation of the high technical level, manufactured in the Russian Federation from domestic raw materials: foams FRV-1, FPBM, FL1, Vilares [13]; compositional materials: PSF-VNIIST, perlite-foam, keramzitphenolic-foam and perlitofosfogel $[4,5,9]$.

\footnotetext{
* Corresponding author: stefurak@yandex.ru
} 
As a result of the analysis and synthesis of a set of elementary functions, a number of hypothetical compositions were created, which were then realized in laboratory and experimental-industrial conditions. Optimization of compositions was carried out by simplex planning [6]. It was found that the foam plastic capable to work in the panel as a heater and facing and decorative material can be only of composite type [1].

We have developed a new type of composite polyfoam (PP), has a pronounced integral structure: the density increases from center to surface due to the formation of a hard crust [21-22].

This paper presents the composition of the new material, the technology of its production, as well as the mathematical model of the mechanism of formation of strong crusts in its surfaces.

\section{Materials used for polyfoam PP}

2.1 As a binder for PP polyfoam used resin FRV-1A, which in its properties corresponds to TU6-05-1104-78.

Phenol-formaldehyde resin FRV-1A is a homogeneous mixture of neutralized aqueous solution of the primary products of alkaline condensation of phenol and formaldehyde, which introduced surface-active substance OP-7 or OP-10 and aluminum powder in the following ratio in parts by weight:

$\begin{array}{ll}\text { Resin FRV-1A } & -100 \\ \text { Aluminum powder } & -1 \\ \text { OP -7 (OP -10) } & -3\end{array}$

2.2 The dynamic viscosity $\eta,\left(N \cdot s / m^{2}\right)$ of the polymer FRV-1A is shown in Table 1.

Table 1. The dynamic viscosity of the polymer FRV-1A

\begin{tabular}{|c|c|c|c|c|c|c|c|}
\hline № parties & 20 & 25 & 30 & 35 & 40 & 45 & 50 \\
\hline 1 & 1.80 & 1.58 & 1.01 & 0.65 & 0.43 & 0.28 & 0.18 \\
\hline 2 & 3.52 & 2.10 & 1.25 & 0.82 & 0.59 & 0.38 & 0.23 \\
\hline
\end{tabular}

2.3 Product VAH-3 - acid poly-dimensional type, representing an aqueous solution of the condensation product of sulfophenyl urea and formaldehyde in a mixture with phosphoric acid. VAH -3 must comply with the requirements of TU 6-05-1116-78.

2.4 The polystyrene shall conform to the requirements of GOST 6-05-202-83. Bulk weight of polystyrene granules is not more than $15 \mathrm{~kg} / \mathrm{m}^{3}$.

2.5 Exfoliated vermiculite, free-flowing granular material flake structure obtained by firing natural gidratirovannykh micas. Vermiculite the chemical composition is a silicate of magnesium and iron. Vermiculite according to GOST 12865-67 is heterogeneous in composition and has an ellipsoidal configuration, its flatness $2,52 \cdot 10^{3}-2,86 \cdot 10^{3} \mathrm{~kg} / \mathrm{m}^{3}$.

2.6 Flotation-oxal (flotoreagent-oxal, TU 38.103-249-80) must match the TU 38.103429-83. Flotation reagent-oxal is a byproduct of isoprene production brought to the necessary condition through the stage of dimethyldioxane production. Introduction to the composition of the flotation agent-oxal allows you to change the structure of the polyfoam and improve its physical and mechanical properties. 


\section{The composition of polyfoam PP}

As a result of experimental studies based on simplex planning [6], [15], the composition of PP was developed. The composition of the polyfoam \% mass:

phenol-formaldehyde resin

granules of polystyrene foam

flotation reagent-oxal

vermiculite

the product of condensation of sulfadimidine with

formaldehyde and orthophosphoric acid (VAH-3)
$-50-57$

$-3-5$

$-2-6$

$-26-31$

- the rest

\section{Technological parameters production polyfoam PP}

The main technological parameters of production polyfoam PP were worked out in laboratory conditions [11]. The process of manufacturing PP is to prepare a reaction-active composition (mixing resin FRV-1A, flotation agent-oxal and foaming-curing agent VAH3 ), mixing it with fillers (polystyrene and vermiculite) and laying the thermal insulation mass in the form. Half the volume of the form is filled with prepared reaction-active composition. Foaming-curing agentVAH-3 and aluminum powder enter into an exothermic reaction. In the form of foaming and curing of the heat-insulating mass (formation of polyfoam). The volume of the finished polyfoam twice the volume of the original composition. The wall forms installed pressure gauge. During reaction of the foaming mold wall are under the pressure of $0-0.06 \mathrm{MPa}$.

Process of production PP (TU 102-433-87) is characterized by the following parameters, it is shown in Table 2.

Table 2. Parameters characterizing process of production PP.

\begin{tabular}{|l|l|}
\hline temperature of the product of VAH $-3,{ }^{\circ} \mathrm{C}$ & $20-22$ \\
\hline temperature of resin FRV-1A and flotation agent-oxal, ${ }^{\circ} \mathrm{C}$ & $20-25$ \\
\hline room temperature, ${ }^{\circ} \mathrm{C}$ & $18-20$ \\
\hline form temperature, ${ }^{\circ} \mathrm{C}$ & $30-40$ \\
\hline time of mixing and laying the mixture into the mold, $\mathrm{S}$ & $120-180$ \\
\hline curing time of not less than, $C$ & 780 \\
\hline the maximum temperature of the exothermic reaction, ${ }^{\circ} \mathrm{C}$ & $100-105$ \\
\hline the operating pressure during foaming, $\mathrm{MPa}$ & 0.06 \\
\hline
\end{tabular}

\section{Mathematical model of the mechanism of formation hard crusts on the surfaces of the polyfoam integrated structure}

Formation of crusts on the surfaces of the polyfoam causes its high mechanical properties. Consider the mechanism of formation of these crusts.

The reactive composition merges into a mixer, where it is mixed with granules of polystyrene and vermiculite. From the mixer activated heat-insulating mass is discharged into the cassette and leveled. In the period of time from 180 to 600 seconds, the foaming reaction of the polyfoam occurs, during which hydrogen is released. Vermiculite particles can move in a liquid medium only in those first five minutes after the start of the foaming reaction, when the mixture is in a liquid state, then the foam is cured. 
PP composition includes flotation reagent-oxal, which reduces the wetting angle of vermiculite with phenolformaldehyde resin by 2 times (from $30^{\circ}$ to $14^{\circ}$ ). More than 30 special additives were experimentally investigated, and only the flotation reagent-oxal observed selective property of floating vermiculite particles in the mixture to the surface. We assume that with the reduction of the vermiculite wetting angle by resin, the viscosity of the medium is equal to the viscosity of the liquid phase of the foaming system. There is an exothermic reaction, the temperature of the composition increases to $100{ }^{\circ} \mathrm{C}$. Already at $30^{\circ} \mathrm{C}$, the viscosity of the polymer FRV-1A is equal to $1.1 \mathrm{~Pa} \cdot \mathrm{s}$.

The multiplicity of the foaming reaction mixture equal to 20 . The gas bubbles released during the reaction join the vermiculite particle, an effective particle is formed, the volume of which significantly exceeds the volume of the vermiculite particle (the volume of the PP composition is doubled, which is due to the bubbles of the released gas). The material becomes porous. Microscopic analysis confirms this hypothesis.

The mass of the effective particle is almost equal to the mass of the vermi-culite particle:

$$
\frac{\rho_{\mathrm{g}}}{\rho_{b}}=\frac{1.1}{2.86 \cdot 10^{3}}=3.85 \cdot 10^{-4},
$$

where $\rho_{v}$ - the density of vermiculite,

$$
\rho_{g} \text { - the density of the gas. }
$$

It is required to determine the mode of motion of the effective particle located in the activated mixture, it is shown in Figure 1.

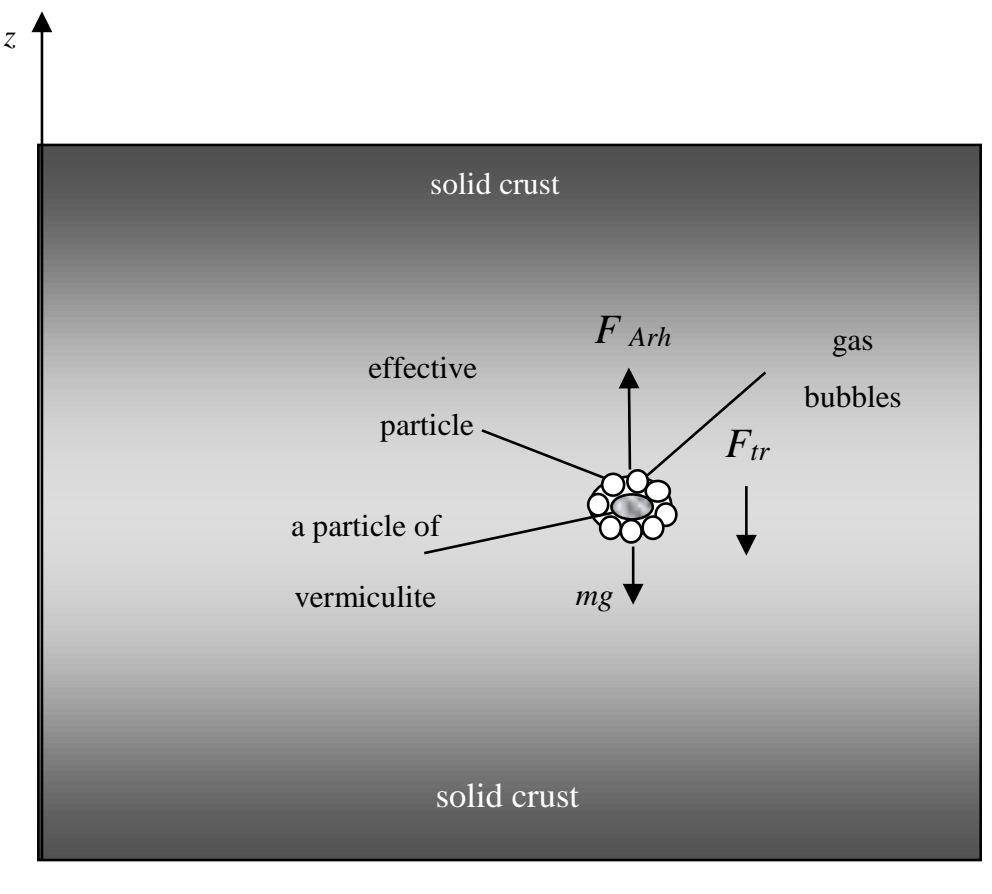

Fig. 1. Effective particle in the activated mixture. 
The mode of movement of a single particle in reaction.

$$
m_{p} \frac{d v_{p}}{d t}=F_{A r h}-F_{t r}-m_{p} g,
$$

where $m_{p}$ - the mass of the particle,

$v_{p}$ - the speed of movement of the particle,

$\frac{d v_{p}}{d t}$ - acceleration of particle,

$F_{A r h}$ - the lifting force of Archimedes,

$F_{t r}$ - the force of resistance to motion of a particle [2].

In this case, in view of the small differences in the velocity of the interfacial surface of the carrier fluid, the reactive force will be neglected [3].

In this case, the particle mass $m_{p}=\frac{4}{3} \pi \cdot R_{p}^{3} \rho_{p}$ is a variable, because it changes in time. Then

$$
\frac{d m_{p}}{d t}=J
$$

where $J$ - is the rate of change of particle mass in time.

The speed of the particle movement is given by the equation

The resistance force

$$
\frac{d z_{p}}{d t}=v_{p}
$$

$$
F_{t r}=\frac{1}{2} C_{d} \rho_{l} v_{p}^{2} \frac{\pi \cdot d_{p}^{2}}{4}=-3 \pi \mu_{l} \rho_{l} d_{p} v_{p},
$$

where $C_{d}$ - the coefficient of resistance to movement, taking into account the influence of the mass transfer process, which in accordance with the law of Stokes is equal to $24 / R e$.

The change of free volume, which causes a change in the pressure in the mixture, is described by the equation

$$
d V_{g}=N \cdot d v_{p}
$$

where $N$ - is the number of particles in the liquid (it can be found through the initial

concentration of the substance in the mixture),

$V_{g}$ - the volume of the gas released during the reaction.

Then the pressures in the mixture can be expressed

$$
P V_{g}=u_{g} R_{\mathrm{g}} T_{\mathrm{g}}
$$

where $u_{\mathrm{g}}$ - the rate of change in the mass of gas in time

$R_{\mathrm{g}}$ - gas constant.

The influence of chemical reactions on the dynamics of the rise of particles surrounded by a gas bubbles [7] is taken into account by Archimedes ' force, which is described by the equation:

$$
F_{A r h}=m_{p} g \frac{\rho_{l}}{\rho_{p}},
$$

where $\rho_{l}$ - the density of the environment,

$\rho_{p}$ - the density of the particle. 
Vermiculite particle at a temperature of $20-105^{\circ} \mathrm{C}$ does not react with the components of the mixture. The change in the volume of the effective particle is due to gas. Therefore, the rate of change in the mass of the effective particle in time $J$ is close to zero:

$$
J \approx 0 \text {. }
$$

The time interval is small and therefore, the filtering effect can be not taken into account. The effective particle in the first approximation is considered to be non-reactive.

Solution of equation (1), with the initial condition that at $t=180 \mathrm{~S}$ the particle is still stationary

$$
v_{p}(180)=0 \text {, }
$$

defines the speed at which the particle moves:

$$
v_{p}=\frac{m g}{3 \pi d \mu \rho_{l}}\left(\frac{\rho_{l}}{\rho_{p}}-1\right) \cdot\left(1-e^{3 \pi d \mu \rho_{l}(180-t)}\right) .
$$

Then the distance run by the effective particle is described by the equation:

$$
\begin{gathered}
S(t)=\frac{m g}{3 \pi d \mu \rho_{l}} \cdot\left(\frac{\rho_{l}}{\rho_{p}}-1\right) . \\
\left(T-180+\frac{1}{3 \pi d \mu \rho_{l}}\left(e^{3 \pi d \mu \rho_{l}(180-T)}-1\right)\right) .
\end{gathered}
$$

Vermiculite according to GOST 12865-67 is heterogeneous in composition and has an ellipsoidal configuration. The density of the vermiculite $2,52 \cdot 10^{3}-2,86 \cdot 10^{3} \mathrm{~kg} / \mathrm{m}^{3}$.

5.1. We study the behavior of a large fraction and determine the path that passes a large effective particle.

Let the average particle size vermiculite: $d_{1}=0.7 \cdot 10^{-2} ; d_{2}=0.5 \cdot 10^{-2} ; d_{3}=0.3 \cdot 10^{-2} \mathrm{~m}$. The mass of the particles of vermiculite:

$$
M=\frac{4 \pi}{3} \rho \cdot \frac{d_{1}}{2} \cdot \frac{d_{2}}{2} \cdot \frac{d_{3}}{2} ; \mathrm{M}=1.39 \cdot 10^{-4}-1.84 \cdot 10^{-4} \mathrm{~kg} .
$$

Then: the mass of the effective particle, which is determined by the mass of the vermiculite, is equal $1.4 \cdot 10^{-4}-1.6 \cdot 10^{-4} \mathrm{~kg}$. The density of the air is $1.2-0.95 \kappa 2 / \mathrm{m}^{3}$. The density of the resin FRV-1A $1.2 \cdot 10^{3} \mathrm{~kg} / \mathrm{m}^{3}$. The density of the product of VAH-3 $1.25 \cdot 10^{3} \mathrm{~kg} / \mathrm{m}^{3}$.

5.1.1. The ratio of the effective particle to volume of the vermiculite particle is equal to 2.96 , the density of effective particles $8.5 \cdot 10^{2}-9.6 \cdot 10^{2} \mathrm{~kg} / \mathrm{m}^{3}$. From (2) we obtain that the effective particle for $40 \mathrm{~s}$ after the beginning of foaming will pass a distance of 0.18 $0.25 \mathrm{~m}$.

5.1.2. The ratio of the effective particle volume to the volume of the ver miculite particle is 1.54 , then: the density of the effective particle is $1.64 \cdot 10^{3}-1.86 \cdot 10^{3} \mathrm{~kg} / \mathrm{m}^{3}$. Get that distance, which will be effective particle for 40 after the beginning of foaming negative: $-0.13-(-0.25) m$. That is - she'll come down.

Thus, the bulk of vermiculite a minute after the beginning of foaming will be in the areas of formation of foam crusts. Microscopic analysis of the foam of PP showed that only $12-18 \%$ vermiculite is distributed in the middle part of the product. 


\section{The physical and mechanical properties of polyfoam PP}

Density cover polyfoam PP is over $200 \mathrm{~kg} / \mathrm{m}^{3}$, and the middle part up $80 \mathrm{~kg} / \mathrm{m}^{3}$. The limit of strength at punching of the polyfoam PP by the rod diameter 10 reaches $3.5 \mathrm{MPa}$, which is twice higher than that of the polyfoam FRP-I density $80 \mathrm{~kg} / \mathrm{m}^{3}$.

\section{Conclusions}

The polyfoam PP, which is used for insulation of roofing panels, BKU panels with an inner non-metallic layer, is obtained. Such panels allow to break bridges of cold in butt joints that improves thermophysical characteristics, reliability and durability of the building as a whole

On the basis of the developed mathematical model it is possible to predict the properties of PP foam plastic.

\section{References}

1. B. Stefurak, L. Stefurak. Construction of pipelines, 10, 22-24 (1978)

2. L. Landau, E. Lifshits. Fizmatlit. Theoretical physics. Hydrodynamics, 6, 736 (2001)

3. R. Nigmatulin. Nauka. Dynamics of multiphase media, 2, 359 (1987)

4. N. Makridin, Structure formation and structural strength of cement composites. MGSU. 152 (2013)

5. L. Dvorkin. Calculated prediction of properties and design of concrete compositions, Infra-Engineering, 386 (2017)

6. Y. Adler, Y. Granovsky, Methodology and practice of planning experiment in Russia. MISIS, 182 (2016)

7. D. Labuntsov, V. Jagow. The Mechanics of two-phase systems. MPEI Publishing house, 384 (2016)

8. V. Lisyansky, P. Blinov. Application of new materials in the construction of a fortification. SPbGPU, 78 (2015)

9. N. Makridin, E. Korolev, Structure formation and structural strength of cement composites. MISI-MGSU, 152 (2013)

10. V. Sokov, Creation of refractory concretes and heat-insulating materials with high temperature resistance: monograph. MISI-MGSU, 288 (2015)

11. A. Tolstoy, V. Lesovik, Technological processes and equipment of enterprises of building materials. Lan, 336 (2015)

12. V. Pakhomov, V. Fedorov, A. Gluzman, B.Ivanov. Telemetry plays a Central role in the control of thermal properties of protective and insulating structures. Naukova Dumka, 33-35 (1990)

13. TU 6-05-221-244-72. Foam stamps of Vilares-5 and glassware. 45 (1972)

14. V. Bindiganavile, N. Banthia, B. Aarup, ACI Materials Journal, 99 (6), 543-548 (2002)

15. V. Vorobyev, B. Stefurak, L. Stefurak, Stroitelnie materiali, 9, 25-26 (1976)

16. Hangelgrock P. Holland, Bouwwereld, 24, 18-20 (1986) 
17. V. Fedotkin., A. Aizen, Asymptotic methods in problems of heat and mass transfer. Higher school, 584 (1972)

18. K. Zatsepin, V. Shaposhnikov. Construction of pipelines, 4, 18-24 (1973).

19. Technology of heat-insulating materials and products. Higher school. (1989)

20. T. Quinn. Temperature. Mir, 447 (1985)

21. M.R. Petrichenko, D.V. Nemova, E.V. Kotov, D.S. Tarasova, V.V. Sergeev, Mag. Civ. Eng., 77 (1), (2018)

22. K. Strogonov, A. Fedyukhin, T. Stepanova, O. Derevianko, Adv. Intell. Sys. Comput., 692, (2018) 